\title{
A Network of Type III Bricard Linkages
}

\author{
Shengnan Lu \\ Robotics Institute \\ Beihang University \\ Beijing, China 100191 \\ Email: Ivshengnan5@gmail.com
}

\author{
Xilun Ding \\ Robotics Institute \\ Beihang University \\ Beijing, China 100191 \\ Email: xlding@buaa.edu.cn
}

\author{
Dimiter Zlatanov \\ PMAR Robotics \\ University of Genoa \\ Genoa, Italy 16145 \\ Email: zlatanov@dimec.unige.it
}

\author{
Simon D. Guest \\ Department of Engineering \\ University of Cambridge \\ Cambridge CB2 1PZ, UK \\ Email: sdg@eng.cam.ac.uk
}

\begin{abstract}
Among Bricard's overconstrained 6R linkages, the third type has two collapsed configurations, where all joint axes are coplanar. The paper presents a one-degree-of-freedom network of such linkages. Using the two coplanar states of the constituent Bricard units, the network is able to cover a large surface with a specific outline when deployed, and can be folded compactly into a stack of much smaller planar shapes. Five geometric parameters describing each type III Bricard mechanism are introduced. Their influence on the outline of one collapsed configuration is discussed and inverse calculation to obtain the parameter values yielding a desired planar shape is performed. The network is built by linking the units, either using scissor linkage elements, if the thickness of the panels can be ignored, or with hinged parallelograms, for a thicker material. Two case studies, in which the Bricard network deploys as a rectangle and a regular hexagon, respectively, are presented, validating the analysis and design methods.
\end{abstract}




\section{Introduction}

Deployable mechanisms (DMs), which are capable of large change in their physical size, have many potential applications in areas such as temporary and emergency architecture and space structures, including antennas and telescopes [1-3]. The scissor linkage has been used as a simple basic element in many creative designs of deployable mechanisms, as in the famous Hoberman polyhedral mechanism [4]. Maden has systematically analyzed the behavior of the scissor linkage as a basic unit of deployable mechanisms with different expansion patterns [5]. Yang et al. construct a series of 2-DOF planar translational DMs based on the scissor linkages [6,7]. Recently, in search of good stiffness and high expansion to package ratio, more attention has been paid to spatial over-constrained linkages as possible building units for the design of deployable mechanisms. Chen et al. have proposed several mobile assemblies which are composed of the Bennett linkages, the Myard linkages, or the Bricard linkages [8,9]. Qi et al. have built a prototype of DM based on the Myard linkages [10]. Lu et al. present a network of Bennett linkages which can be deployed to approximate a cylindrical surface [11].

Bricard linkages are based on Bricard Octahedra: these self-intersecting, zerointernal-volume polyhedra have a single finite mechanism $[12,13]$. There are three types of Bricard octahedra. The first is line-symmetric and the second is planesymmetric; the third type of Bricard octahedra is however somewhat less simple. It has two collapsed configurations (i.e., states where all the revolute joints are coplanar), so the type III Bricard linkage is also named doubly collapsible octahedral linkage [14]. Mechanisms based on the first two Bricard octahedra are well-studied [15-19], but the type III Bricard linkage has attracted less attention compared to other Bricard types [14,20,21].

Due to the ability to collapse in two ways, a network of type Bricard III linkages can cover a large surface with a specific outline when deployed, and can be folded compactly into a stack of much smaller planar shapes. In this study ${ }^{1}$, we discuss how to build and shape such a network by linking Bricard linkages with either the same or different geometric parameters.

The paper is organized as follows. In the following section, the type III Bricard linkage is reviewed and parameterized. Then, the two collapsed configurations of the mechanism are analyzed, including the influence of the parameters on the planar shape. Next, the range constraints of the parameters are discussed. Inverse calculation to obtain the parameter values of a specific deployed configuration is derived. Then, two methods of connecting linkages are proposed to build the network. Two case studies are performed to obtain different deployed shapes, and each is validated by a simulation.

\footnotetext{
${ }^{1}$ Preliminary versions of parts of this work were presented in August 2015 at ASME-IDETC (Paper 47139) in Boston, Massachusetts, and in [22].
} 


\section{The Type III Bricard Linkage}

\subsection{Geometric Construction}

The Bricard linkage $A B C A^{\prime} B^{\prime} C^{\prime}$ in Fig. 1, is of type III with two collapsed states. The linkage can be constructed as follows: draw two concentric circles of arbitrary radii; choose two arbitrary points $A$ and $A^{\prime}$ outside of the larger circle; construct the tangents from $A$ and $A^{\prime}$ to the circles and determine their intersections $B, B^{\prime}, C$ and $C^{\prime}$. The lines $B C, B^{\prime} C^{\prime}, B^{\prime} C$ and $B C^{\prime}$ will be tangent to a third concentric circle with radius $r_{t}$. Then, the six triangles $A B C, B C A^{\prime}, C A^{\prime} B^{\prime}, A^{\prime} B^{\prime} C^{\prime}, B^{\prime} C^{\prime} A$, and $C^{\prime} A B$, taken in that cyclic order, hinged at their common edges, constitute a deformable six-plate linkage with 1 dof [23].

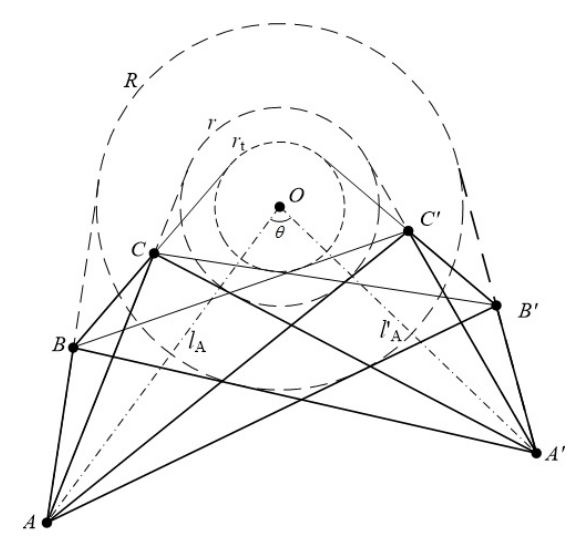

Fig. 1. Construction of a Bricard linkage

The six triangles in fact define a deformable octahedron: the two remaining (virtual) faces are the triangles $A C B^{\prime}$ and $A^{\prime} C^{\prime} B$, whose shape is constant during the movement. However, if they are physically part of the linkage, link interference is unavoidable. Furthermore, any six of the eight faces can be hinged together, generating an equivalent motion of the octahedron.

\subsection{Parametrization of the Type III Bricard Geometry}

The construction in Fig. 1, can be described by the radii of the two circles and the positions of points $A$ and $A^{\prime}$. We denote the radii of the larger and smaller concentric circles by $R$ and $r$, respectively. The lengths of $O A$ and $O A^{\prime}$ are $l_{A}$ and $l_{A}^{\prime}$. The fifth parameter, is the angle between $O A$ and $O A^{\prime}$, denoted by $\theta$. A Bricard linkage will be decided by those five scalars, $R, r, l_{A}, l_{A}^{\prime}$, and $\theta$. One of the five parameters controls the scale of the Bricard linkage; the other four decide the collapsed configuration, which determines the shape and movement of the Bricard linkage. 


\section{The Deployed Shape}

The two collapsed configurations of the type-III Bricard linkage will be used for the compact and deployed state of the proposed mechanisms. The shape of the planar area covered or enclosed by the physical links in these states depends on the geometric parameters of the linkage, identified above. This section analyzes this dependence.

\subsection{The Two Collapsed Configurations}

Figure 1 shows one of the collapsed configurations of the type III Bricard linkage, used as its compacted state. The six revolute-joint axes are along $A B, B C, C A^{\prime}, A^{\prime} B^{\prime}, B^{\prime} C^{\prime}, C^{\prime} A$, linked in this order, Fig. 2. The resulting $6 \mathrm{R}$ loop is mobile and can be continuously transformed into another, deployed state, where joint axes are coplanar again.

It can be noted that adjacent axes intersect, i.e., link lengths along the common normals are zero. As mentioned in Sec 2.1, the physical links are triangles with vertices in the axes-intersection points: $A B C, B C A^{\prime}, \ldots, C^{\prime} A B$.

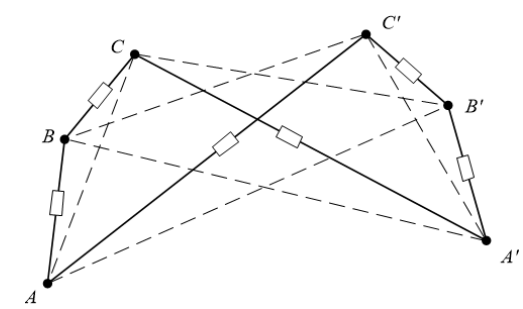

Fig. 2. Schematic of the type III Bricard linkage

An example of the two collapsed configurations of one linkage is shown in Fig. 3. In the following we focus on the geometric shape and dimensions of the polygon defined as a convex envelope of the six points where the joint axes intersect. This convex shape is a hexagon in Fig. 3(a). In Fig. 3(b), the outline is a quadrangle with vertices, $A_{*}, C_{*}, A_{*}^{\prime}$, and $C_{*}^{\prime}$.

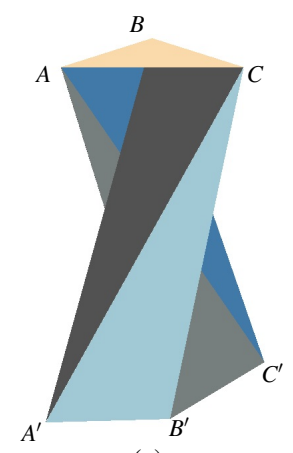

(a)

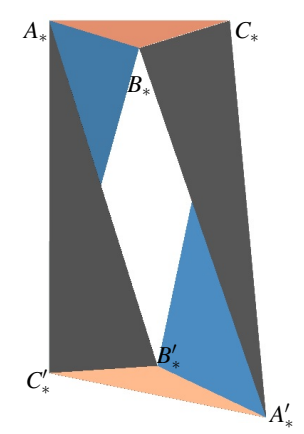

(b)

Fig. 3. Two collapsed configurations of the type III Bricard 


\subsection{The Deployed Quadrangle}

It is important to understand how the geometry of the Bricard linkage, i.e., the choice of the five parameters identified in the preceding section, defines the shape of the quadrangle $A_{*} C_{*} A_{*}^{\prime} C_{*}^{\prime}$.

In Fig. 1, the angles are named as follows, $\angle B A B^{\prime}=\beta_{A}, \angle B A^{\prime} B^{\prime}=\beta_{A}^{\prime}$. As $A B$ and $A B^{\prime}$ are tangent to the big circle, Fig. 1,

$$
\angle O A B=\angle O A B^{\prime} \Rightarrow \beta_{A}=2 \alpha_{A 1}=2 \arcsin \frac{R}{l_{A}}
$$

Lines $A C$ and $A C^{\prime}$ are tangent with the small circle, hence

$$
\angle O A C=\angle O A C^{\prime}
$$

Therefore,

$$
\angle B A C=\angle B^{\prime} A C^{\prime}
$$

In Fig. 3(a), it can be seen that $\angle B A B^{\prime}=\angle B^{\prime} A C^{\prime}+\angle B A C^{\prime}$. Similarly, from Fig. 3(b), $\angle C_{*} A_{*} C_{*}^{\prime}=\angle B_{*} A_{*} C_{*}+\angle B_{*} A_{*} C_{*}^{\prime}$. From (3),

$$
\angle C_{*} A_{*} C_{*}^{\prime}=\beta_{A}=2 \arcsin \frac{R}{l_{A}}
$$

where $\angle C_{*} A_{*} C_{*}^{\prime}$ is in the deployed configuration.

Obviously, each rigid triangle remains congruent in both collapsed configurations, and throughout the motion. 
Similarly,

$$
\angle C_{*} A_{*}^{\prime} C_{*}^{\prime}=\beta_{A}^{\prime}=2 \arcsin \frac{R}{l_{A}^{\prime}} .
$$

Because of symmetry, it is sufficient to consider the case $l_{A} \geqslant l_{A}^{\prime}$, which implies $\beta_{A} \leqslant \beta_{A}^{\prime}$.

Since the edge length stays constant, $A C, A^{\prime} C, A C^{\prime}, A^{\prime} C^{\prime}$ are used to describe the edges of the quadrangle. They are the tangent lines from $A$ and $A^{\prime}$ to the small circle, Fig. 4.

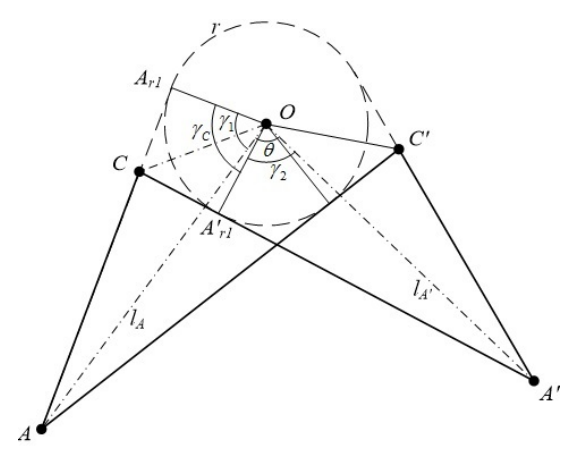

Fig. 4. Dimensions of the quadrangle

We denote:

$$
\left\{\begin{array}{l}
\gamma_{1}=\arcsin \frac{r}{l_{A}} \\
\gamma_{2}=\arcsin \frac{r}{l_{A}^{\prime}}
\end{array}\right.
$$

$$
\gamma_{c}=\gamma_{1}-\gamma_{2}+\theta
$$

$A_{r 1}$ and $A_{r 1}^{\prime}$ are the points of tangency from $A$ and $A^{\prime}$ to the small circle. So, 


$$
\left|A A_{r 1}\right|=\sqrt{l_{A}^{2}-r^{2}}
$$

$$
\left|C A_{r 1}\right|=r \tan \frac{\gamma_{c}}{2}=r \tan \frac{\arcsin \frac{r}{l_{A}}-\arcsin \frac{r}{l_{A}^{\prime}}+\theta}{2}
$$

\section{Therefore,}

$$
|A C|=\sqrt{l_{A}^{2}-r^{2}}-r \tan \frac{\arcsin \frac{r}{l_{A}}-\arcsin \frac{r}{l_{A}^{\prime}}+\theta}{2}
$$

$$
\left|A^{\prime} C\right|=\sqrt{l_{A}^{\prime 2}-r^{2}}+r \tan \frac{\arcsin \frac{r}{l_{A}}-\arcsin \frac{r}{l_{A}^{\prime}}+\theta}{2}
$$

Similarly,

$$
\left|A C^{\prime}\right|=\sqrt{l_{A}^{2}-r^{2}}+r \tan \frac{\arcsin \frac{r}{l_{A}^{\prime}}+\arcsin \frac{r}{l_{A}}+\theta}{2}
$$




$$
\left|A^{\prime} C^{\prime}\right|=\sqrt{l_{A}^{\prime 2}-r^{2}}-r \tan \frac{\arcsin \frac{r}{l_{A}^{\prime}}+\arcsin \frac{r}{l_{A}}+\theta}{2}
$$

It can be found from (10)-(13) that

$$
|A C|+\left|A^{\prime} C\right|=\left|A C^{\prime}\right|+\left|A^{\prime} C^{\prime}\right|,
$$

which means that the shape of the formed quadrangle cannot be a trapezoid.

The correspondence between the shape of quadrangle $A_{*} C_{*} A_{*}^{\prime} C_{*}^{\prime}$ and the 5 parameters of the type III Bricard linkage is a one-to-many mapping. Multiple sets of of parameters define the same outline, with different locations of $B$ and $B^{\prime}$.

\subsection{Parameter ranges}

The construction of the Bricard linkage imposes bounds for the value of each parameter. The mechanism cannot be constructed when a parameter is out of its domain of definition. Special cases occur when a parameter has an extreme value.

For example, when $\theta=0, l_{A}=l_{A}^{\prime}$, and $A$ is coincident with $A^{\prime}$. The outline of the mechanism in the compact configuration becomes a pentagon. The locations of $B$ and $B^{\prime}$ are no longer unique, as they can be any points on the tangent lines. The result, shown in Fig. 5, is the construction of a crinkle mechanism, used by Connelly in the development of the first truly flexible polyhedron [24].

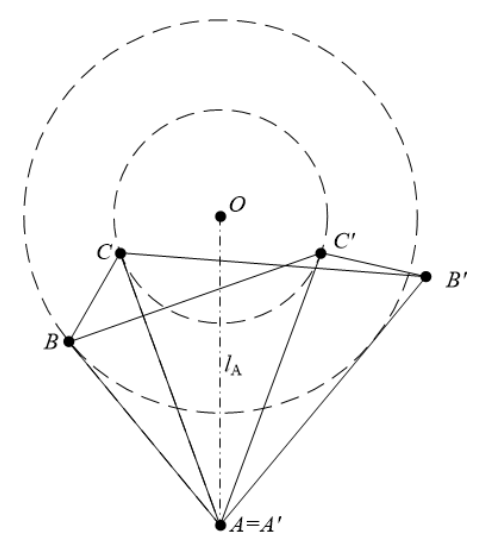

Fig. 5. Construction of a crinkle mechanism 
Figure 6 displays the movement between two coplanar configurations of a crinkle mechanism. In this example, $A B=$ $A^{\prime} B^{\prime}, A C=A^{\prime} C^{\prime}$.

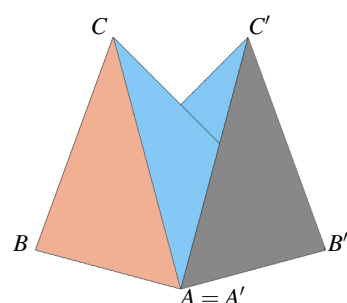

(a)

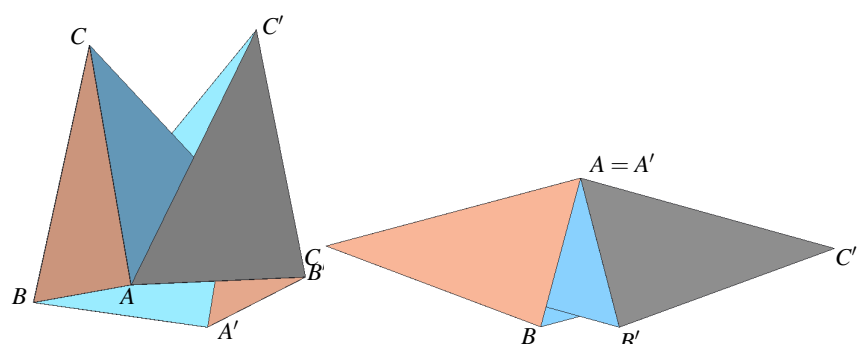

(b)

(c)

Fig. 6. Movement process of a crinkle linkage

There are other similar cases, for instance when $r=R$, and $B$ is coincident with $C$. In the following, we will restrain the parameters to be inside their domains of definition to avoid those special cases. The ranges are:

$$
\left\{\begin{array}{l}
0<r<R \\
l_{A}>R \\
\theta>0
\end{array}\right.
$$

In the construction of the Bricard linkage, the possible locations of $B$ can be divided into 3 classes: $B$ between the tangent point and $A, B$ coinciding with $A$, and $A$ between the tangent point and $B$.

The latter case, can be seen as exchanging $A$ and $B, A^{\prime}$ and $B^{\prime}$ in Fig. 1 . The mechanism can be equivalent to another Bricard linkage, for which $B$ is between $A$ and the tangent point, with different $l_{A}, l_{A}^{\prime}$ and $\theta$. Therefore, there is another inequality condition on those four parameters. Their relationship in the limited position is shown in Fig. 7, where $B$ and $A$ coincide, and so do $B^{\prime}$ and $A^{\prime}$.

To meet the constraint, $R$ should satisfy

$$
R<\frac{l_{A} l_{A}^{\prime} \sin \theta}{\sqrt{l_{A}^{2}+l_{A}^{\prime 2}-2 l_{A} l_{A}^{\prime} \cos \theta}}
$$




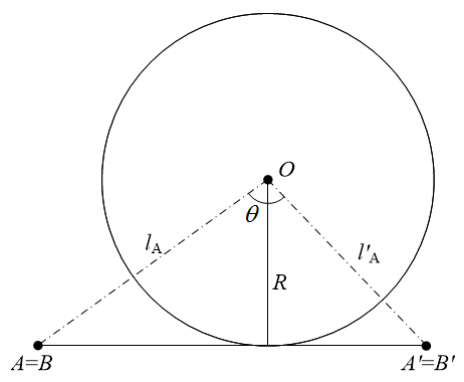

Fig. 7. Limit positions of $A$ and $A^{\prime}$

\subsection{Influence of each parameter}

The shape of the quadrangle is determined by the 5 parameters of the Bricard linkage. Herein we examine the role of each variable. From (4), (5), (10), (11), (12) and (13), the angles are functions of $R, l_{A}$ and $l_{A}^{\prime}$, while the lengths are functions of $l_{A}, l_{A}^{\prime}, r$ and $\theta$. To verify this result, and provide some intuition about the influence of each parameter, we simulate the change of shape when all but one parameters are constant.

Each simulation shows a series of the deployed configurations of the Bricard linkages constructed for incrementally changing values of the chosen parameter. The quadrangle in red is obtained for the minimum value of the variable. Without loss of generality, $A C A^{\prime} C^{\prime}$ is used to described the quadrangle in the following.

To avoid discontinuity of the change of configuration, the range conditions (15) and (16) are respected in all simulations.

In the first simulation, the radius of the big circle, $R$, is variable, while $r=25, \theta=90^{\circ}, l_{A}=80$ and $l_{A}^{\prime}=75$ are fixed. The results are shown in Fig. 8. It can be seen that the edge lengths of the quadrangle stay constant; when $R$ increases, $\angle C A C^{\prime}$ becomes bigger. The simulation result accords with (4) showing that $\angle C A C^{\prime}$ covaries with $R$.

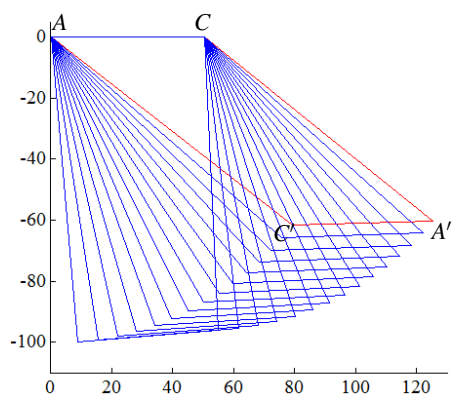

Fig. 8. Influence of $R$ on the collapsed configurations

The variables in the next two illustrations are $\theta$ and $r$, respectively, Fig. 9. Consistent with equations (4) and (5), $\theta$ and $r$ have no influence on any angle of the quadrangle; they only impact the lengths of the edges.

Figure 10(a) and 10(b) show that $l_{A}^{\prime}$ does not alter the angle $\angle C A C^{\prime}$ and $l_{A}$ has no influence on the angle $\angle C A^{\prime} C^{\prime}$. 


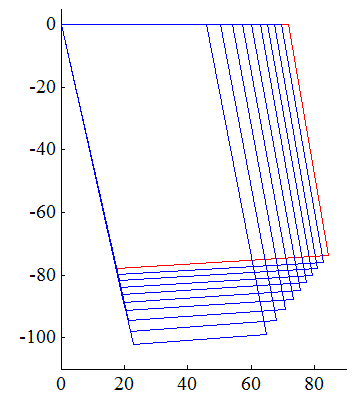

(a) Influence of $\theta$

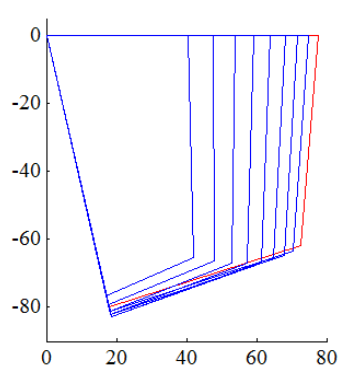

(b) Influence of $r$

Fig. 9. Influence of $\theta$ and $r$ on the collapsed configurations

However, all the edges are affected.

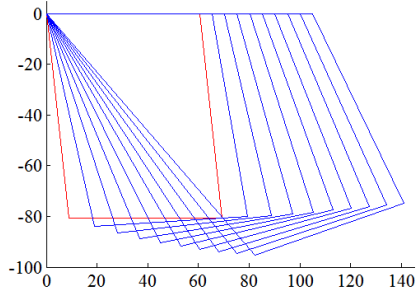

(a)

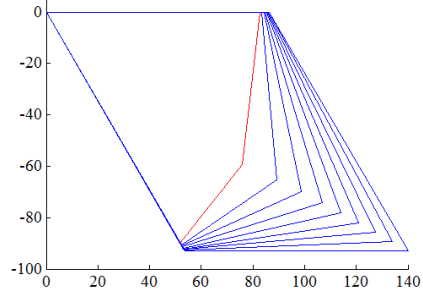

(b)

Fig. 10. Influence of $l_{A}$ and $l_{A}^{\prime}$ on the collapsed configurations

\subsection{Inverse calculation}

In the following, the inverse calculation will be discussed, i.e., the problem of obtaining the parameter values yielding a desired planar shape.

Suppose that shape and size of the quadrangle are known, i.e., $|A C|,\left|A C^{\prime}\right|,\left|A^{\prime} C^{\prime}\right|,\left|A^{\prime} C\right|, \angle C A C^{\prime}=\beta_{A}, \angle C A^{\prime} C^{\prime}=\beta_{A}^{\prime}$ are all given. It can be derived that

$$
\frac{R}{l_{A}}=\sin \frac{\beta_{A}}{2}
$$

$$
\frac{R}{l_{A}^{\prime}}=\sin \frac{\beta_{A}^{\prime}}{2}
$$


Therefore, the ratio between $l_{A}$ and $l_{A}^{\prime}$ is known as $\frac{l_{A}}{l_{A}^{\prime}}=\frac{\sin \left(\beta_{A}^{\prime} / 2\right)}{\sin \left(\beta_{A} / 2\right)}$.

From (10) and (11), we have

$$
\sqrt{l_{A}^{2}-r^{2}}+\sqrt{l_{A}^{\prime 2}-r^{2}}=|A C|+\left|A^{\prime} C\right|=l_{1}
$$

Let $l_{2}=\left|A C^{\prime}\right|-|A C|$. From (12) and (10)

$$
\frac{2 r \sin \theta}{\cos \left(\arcsin \frac{r}{l_{A}}-\arcsin \frac{r}{l_{A}^{\prime}}\right)+\cos \theta}=l_{2}
$$

Therefore,

$$
\arcsin \frac{r}{l_{A}}-\arcsin \frac{r}{l_{A}^{\prime}}=\arccos \frac{2 r \sin \theta-l_{2} \cos \theta}{l_{2}}
$$

Let $l_{3}=\left|A C^{\prime}\right|$, from (12) we have

$$
\sqrt{l_{A}^{2}-r^{2}}+r \tan \frac{\arcsin \frac{r}{l_{A}^{\prime}}+\arcsin \frac{r}{l_{A}}+\theta}{2}=l_{3}
$$

With known three lengths and two angels of the quadrangle, the values of each parameter can be obtained by solving (17)-(22).

A simulation which reveals the correlation among the 5 parameters is performed. The desired quadrangle $A C A^{\prime} C^{\prime}$ is constrained by $|A C|=70,\left|A C^{\prime}\right|=80,\left|A^{\prime} C^{\prime}\right|=70, \beta_{A}=60^{\circ}$, and $\beta_{A}^{\prime}=70.53^{\circ}$. In the simulation, the values of $r$, which 
varies in a range determined by (15) and (16), are also given as input. Values of the other 4 parameters $\left(l_{A}, l_{A}^{\prime}, R, \theta\right)$, corresponding to the desired quadrangle and the given $r$, are calculated by using (18)-(21).

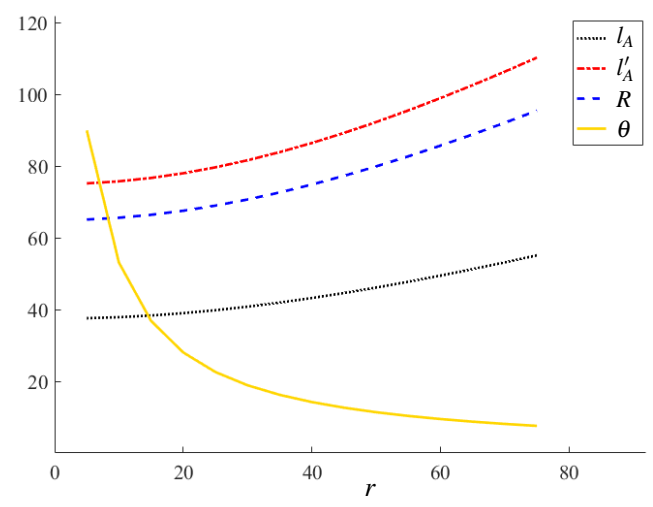

Fig. 11. Simulation of inverse calculation

Simulation results are shown in Fig. 11. It can be seen that except $\theta$ the other three parameters are increased with the increment of $r$. Meanwhile, ratio between $l_{A}$ and $R$ keeps constant which is matched with (17). So does the ratio between $l_{A}^{\prime}$ and $R$. All the parameter sets in Fig. 11 result in the same $A C A^{\prime} C^{\prime}$ quadrangle, the differences are the locations of $B$ and $B^{\prime}$ of the linkage.

\section{Connecting Type III Bricard Linkages}

A deployable network is constructed by linking unit linkages. Two type-III Bricards can be connected using either a scissor likage or a hinged parallelogram. The connecting element depends on whether thickness (of the physical triangular links of the $6 \mathrm{R}$ loop) is negligible.

\subsection{Connection via a scissor linkage}

If the material is very thin, such as in a paper model, the limitation of movement generated by the thickness of the model can be ignored. Under this condition, two Bricard linkage can be linked together by using a common hinge, as shown in Fig. 12(a). A simulated model of the assembly is shown in Fig. 12(b).

By sharing the revolute joint $A^{\prime} C$ and their corresponding panels $A^{\prime} B^{\prime} C$ and $A^{\prime} B C$, two Bricard linkages are connected together, which can also be seen as adding a scissor element between the two Bricards. In the Figure, one common panel is $B C B_{1}^{\prime} A^{\prime}$ and $B_{1} C B^{\prime} A^{\prime}$ is another; the two are hinged along $A^{\prime} C=A_{1}^{\prime} C_{1}$. The angle between these two shared panels is the same for the linkages, and hence they move synchronously. This means that the dof remains when units are combined and therefore the number of addable Bricard linkage is unlimited. 


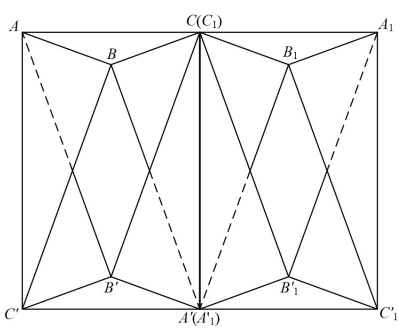

(a)

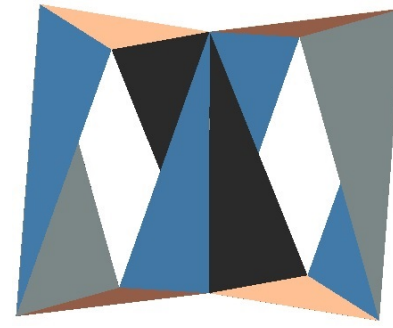

(b)

Fig. 12. Two Bricard linkages linked by scissor linkage

The deployed and compact configurations of such an assembly are shown in Fig. 13. The compact configuration is the same as that of a single Bricard linkage, while the deployed configuration is doubled. The geometric shape of the deployed configuration of the assembly is totally dependent on that of the unit Bricard linkage. Furthermore, the two Bricard linkages are connected by a scissor linkage, which is a parameter-free connection, therefore, the Bricard linkages with different parameters are also able to be connected together. An example of such a case is shown in next section.

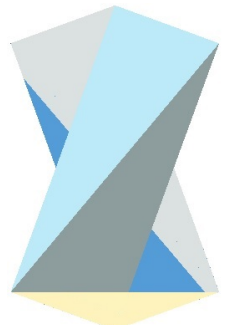

(a)

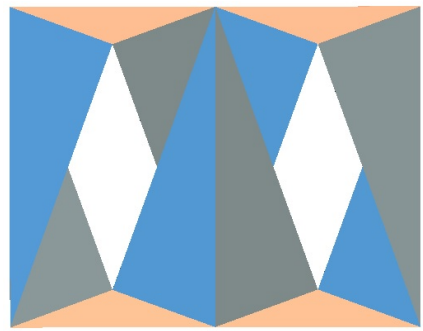

(b)

Fig. 13. The compact and deployed configuration of the network linked with scissor linkage

\subsection{Connection via a parallelogram mechanism}

A hinged parallelogram can be used if the panel thickness cannot be ignored. The parallelogram, which also has 1-dof, plays the same role as the scissor linkage (to connect two adjacent Bricard linkages and maintain the degree of freedom) in the assembly. With the assumption that all the panels have the same thickness, parallelogram mechanisms with the same link lengths are used to construct the network. The working principle of this connection is shown in Fig. 14.

The figure can be seen as a partial top view of the parallelogram mechanism connection, where joints $a$ and $c$ indicate the rotating axes $A^{\prime} C$ and $A_{1}^{\prime} C_{1}$ of the Bricard linkages. The common edges of the corresponding panels are $b$ and $d$. In a parallelogram, $\angle b a d$ is always equal to $\angle b c d$, which makes sure there is a synchronous movement between the two combined Bricard linkages. Without physical link interference, each joint can perform a half-circle rotation.

In the deployed configuration, Fig. 14(b), $a$ and $c$ and the two side surfaces of the panel of the Bricard linkage are 


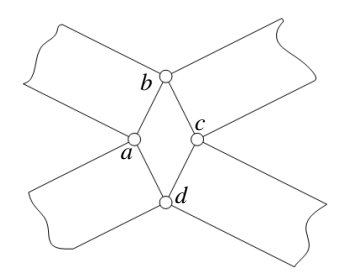

(a)

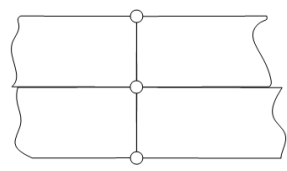

(b)

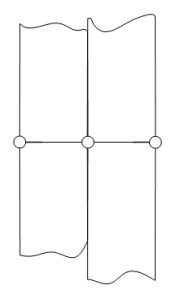

(c)

Fig. 14. Working principle of the parallelogram mechanism

coincident, and the unit linkages are placed side by side. In the compact configuration, Fig. 14(c), $b$ is coincident with $d$, and the units are on top of each other, which guarantees the assembly can be folded without collision, Fig. 15.

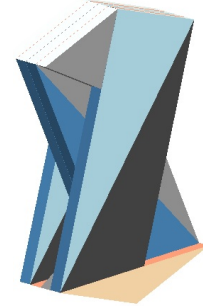

(a)

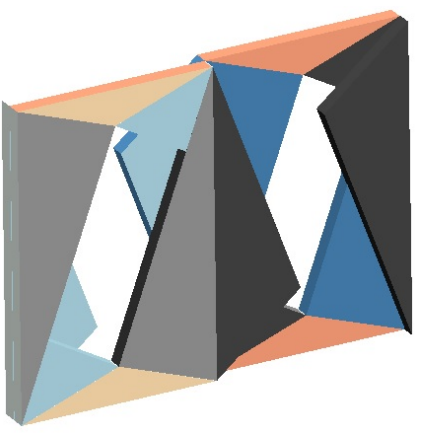

(b)

Fig. 15. Assembly comprised of Bricard linkages and a parallelogram mechanism

In order to avoid collision among the panels of a Bricard linkage, the shapes of the panels have been modified a little, without affecting the outline of the mechanism.

\section{Case Studies}

In this section, two case studies are performed. The Bricard network deploys as a rectangle and a regular hexagon, respectively. To obtain the shapes, different Bricard parameters are adopted. Since one of the variables controls the scale of the Bricard mechanism, the radius of the small circle, $r$, is the same in each case.

\subsection{Case I}

The deployed shape of the network in this case is a rectangle. The Bricard mechanism with a rectangular outline is used as a basic unit in the network. Given $|A C|+\left|A^{\prime} C\right|=\left|A C^{\prime}\right|+\left|A^{\prime} C^{\prime}\right|$, in order to obtain a rectangular $A C A^{\prime} C^{\prime}$, an extra condition should be satisfied in the selected Bricard mechanism, 


$$
\beta_{A}=\beta_{A}^{\prime}=90^{\circ},
$$

which yields $l_{A}=l_{A}^{\prime}=\sqrt{2} R$.

Under the above conditions, $\theta$ determines the length-to-width ratio of the rectangle. When $r=25, R=50$, and $l_{A}=$ $l_{A}^{\prime}=50 \sqrt{2}$, the changing of the rectangle corresponding to different $\theta$ values is shown in Fig. 16.

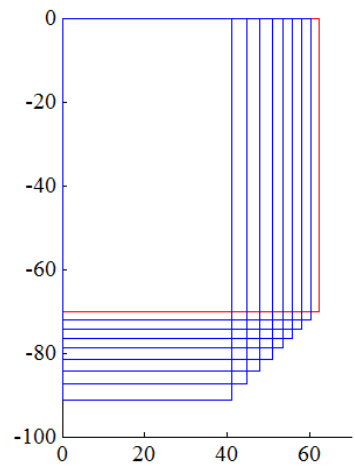

Fig. 16. Formed rectangle corresponding to variable $\theta$

A Bricard mechanism with $\theta=20^{\circ}$ is selected as the unit in the rectangular assembly. The deployment process of a paper model of the Bricard linkage network composed of 3 units is shown in Fig. 17.

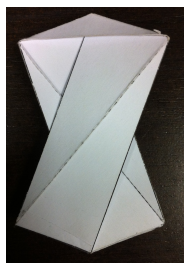

(a)

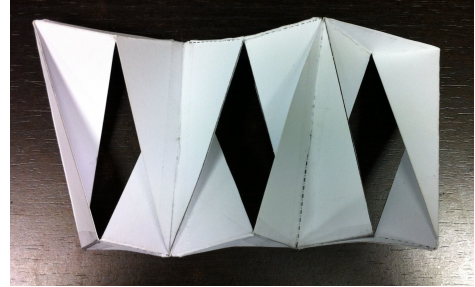

(b)

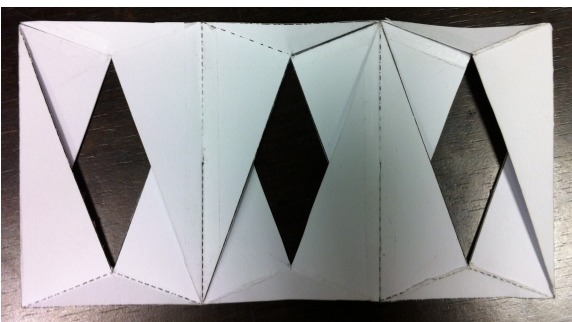

(c)

Fig. 17. Case study I

In the paper model, the thickness of the panel can be ignored. Therefore, the network is constructed by using scissor elements to link the Bricard linkages. In the deployed and compact configurations, all the hinges are almost in the same plane; during the deployment process, the assembly is a spatial mechanism. 


\subsection{Case II}

The network, in this case, is composed of Bricard linkages with different parameters. The outline of the assembly is a regular hexagon. The first Bricard linkage is placed at the corner of the polyhedron, the second one is on the side.

To form a hexagon, the first Bricard linkage should satisfy,

$$
\beta_{A}=90^{\circ} \text {, }
$$

$$
\beta_{C}=90^{\circ}-360^{\circ} / 12=60^{\circ}
$$

Given $r=25, l_{A}=\sqrt{2} R=50 \sqrt{2}$, and $l_{A}^{\prime}=51, \theta=25.41^{\circ}$ is calculated from (21). Therefore, from (12), $\left|A C^{\prime}\right|=69.81$.

The outline of the second Bricard linkage is a rectangle, whose long side is equal to the edge of the first quadrangle, that is $\left|A C^{\prime}\right|=\left|A^{\prime} C\right|=69.81$. With given parameters $r=25$ and $l_{A}=l_{A}^{\prime}=\sqrt{2} R=45 \sqrt{2}$, it is calculated that $\theta=48.63^{\circ}$.

The two unit Bricard linkages are shown in Fig. 18.

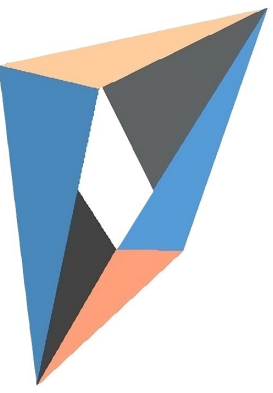

(a)

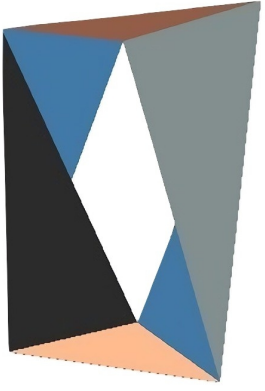

(b)

Fig. 18. Bricard linkages in case study II

By linking 24 linkages of the above two Bricard geometries, a CAD model is built. The model is simulated and the moving process is shown in Fig. 19. Simulation of the assembly proves that a Bricard network can be constructed either using identical or different units. 


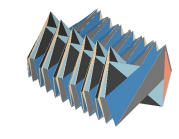

(a)

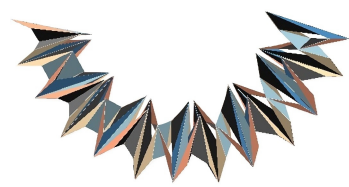

(b)

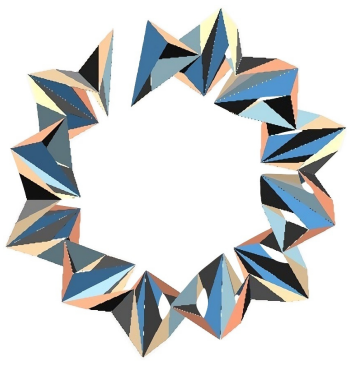

(c)

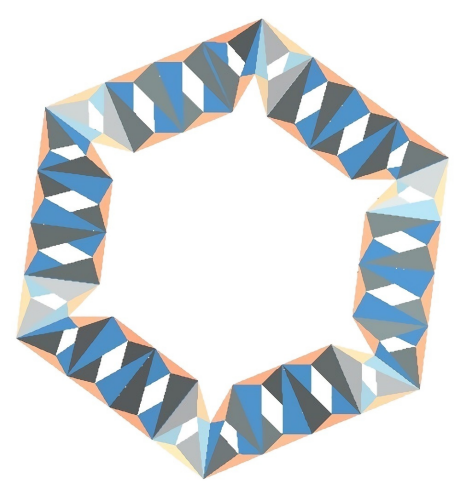

(d)

Fig. 19. Case study II

\section{Conclusion}

This paper presents a novel family of 1-dof networks of type III Bricard linkages. Due to the doubly collapsible feature of the type III Bricard linkage, each such deployable mechanism can cover an arbitrarily large surface with a specific outline when deployed, and can be folded compactly into a stack of much smaller planar shapes. The type III Bricard mechanism is described by five geometric parameters, the relationship among the parameters and the outline of the deployed configuration of the Bricard linkage is discussed. Parameter range constraints for constructing the mechanism are derived. Inverse calculation to obtain the parameter values yielding a desired planar shape is performed. The Bricard network can be built in two ways, linked by scissor linkages, when thickness of the panels can be ignored, or by parallelograms if the material is thick. The proposed method can achieve the proper unit Bricard linkages that could construct a deployable network with specific geometric shape.

Two case studies have been performed, which present the procedure of obtaining a deployed rectangle and regular hexagon, respectively. It is also illustrated that Bricard linkages with different parameters can be linked together. Simulations of the obtained assembly validate the proposed analysis and design methods. The proposed design can be potentially used in the solar array mechanism in a satellite or as a deployable roof of a transportable canopy.

This paper focuses on the Bricard linkage built by the triangular panels in Fig. 1. The outline of the linkage and the network is formed by the vertices of the Bricard linkage. It is known that the structural design of a mechanism may vary its physical shape dramatically. Some possible such variations will be reported in future work.

\section{ACKNOWLEDGEMENT}

This research has been supported by the National Natural Science Foundation of China under Grant 51605011, 51635002(Key Program), and the AUTORECON project funded under the Seventh Framework Program of the European Commission (Col- 
laborative Project NMP-FOF-2011-285189). The authors gratefully acknowledge the supporting agencies.

\section{References}

[1] Escrig, F., Valcarcel, J. P., and Sanchez, J., 1996. "Deployable cover on a swimming pool in Seville". Bulletin of the International Association for Shell and Spatial Structures, 37(1), pp. 39-70.

[2] Zhao, J. S., Wang, J. Y., Chu, F. L., Feng, Z. J., and Dai, J. S., 2012. "Mechanism synthesis of a foldable stair". Journal of Mechanisms and Robotics, 4(1), p. 014502.

[3] Durand, G., Sauvage, M., Bonnet, A., Rodriguez, L., et al., 2014. "Talc: a new deployable concept for a 20-m farinfrared space telescope". In SPIE Astronomical Telescopes+ Instrumentation, International Society for Optics and Photonics, pp. 91431A-91431A.

[4] Hoberman, C., 1991. Radial expansion/retraction truss structures, June 18. US Patent 5,024,031.

[5] Maden, F., Korkmaz, K., and Akgün, Y., 2011. "A review of planar scissor structural mechanisms: geometric principles and design methods". Architectural Science Review, 54(3), pp. 246-257.

[6] Yang, Y., Tian, Y., Peng, Y., and Pu, H., 2017. "A novel 2-DOF planar translational mechanism composed by scissorlike elements". Mechanical Sciences, 8(1), pp. 179-193.

[7] Yang, Y., Peng, Y., Pu, H., and Cheng, Q., 2018. "Design of 2-DOF planar translational mechanisms with parallel linear motion elements for an automatic docking device". Mechanism and machine theory, 121, pp. 398-424.

[8] Chen, Y., 2003. "Design of structural mechanisms". PhD thesis, University of Oxford.

[9] Liu, S. Y., and Chen, Y., 2009. "Myard linkage and its mobile assemblies". Mechanism and Machine Theory, 44(10), pp. 1950-1963.

[10] Qi, X. Z., Deng, Z. Q., Li, B., Liu, R. Q., and Guo, H. W., 2013. "Design and optimization of large deployable mechanism constructed by Myard linkages". CEAS Space Journal, 5(3-4), pp. 147-155.

[11] Lu, S., Zlatanov, D., and Ding, X., 2017. "Approximation of cylindrical surfaces with deployable Bennett networks". Journal of Mechanisms and Robotics, 9(2), p. 021001.

[12] Bricard, R., 1897. "Mémoire sur la théorie de l'octaèdre articulé". Journal de Mathématiques pures et appliquées, pp. 113-148.

[13] Bushmelev, A. V., and Sabitov, I. K., 1991. "Configuration spaces of Bricard octahedra”. Journal of Mathematical Sciences, 53(5), pp. 487-491.

[14] Baker, J. E., 2009. “On the skew network corresponding to Bricard's doubly collapsible octahedron". Proceedings of 
the Institution of Mechanical Engineers, Part C: Journal of Mechanical Engineering Science, 223(5), pp. 1213-1221.

[15] Waldron, K. J., 1969. “Symmetric overconstrained linkages”. Journal of Engineering for Industry, 91(1), pp. 158-162.

[16] Waldron, K. J., 1979. “Overconstrained linkages”. Environment and Planning B: Planning and Design, 6(4), pp. 393402.

[17] López-Custodio, P., Dai, J., and Rico, J., 2018. "Branch reconfiguration of Bricard linkages based on toroids intersections: Plane-symmetric case". Journal of Mechanisms and Robotics, 10(3), p. 031002.

[18] López-Custodio, P., Dai, J., and Rico, J., 2018. "Branch reconfiguration of Bricard linkages based on toroids intersections: Line-symmetric case”. Journal of Mechanisms and Robotics, 10(3), p. 031003.

[19] Feng, H., Chen, Y., Dai, J. S., and Gogu, G., 2017. "Kinematic study of the general plane-symmetric Bricard linkage and its bifurcation variations". Mechanism and Machine Theory, 116, pp. 89-104.

[20] Lu, S., Zlatanov, D., Ding, X., Zoppi, M., and Guest, S., 2015. “Folding type III Bricard linkages”. In Proceedings of the 14th IFToMM World Congress, pp. 455-462.

[21] Lu, S., Zlatanov, D., Ding, X., Zoppi, M., and Guest, S. D., 2016. "Reconfigurable chains of bifurcating type III Bricard linkages". In Advances in Reconfigurable Mechanisms and Robots II. Springer, pp. 3-14.

[22] Lu, S., Zlatanov, D., Ding, X., Zoppi, M., and Guest, S. D., 2015. “A network of type III Bricard linkages”. In ASME 2015 International Design Engineering Technical Conferences and Computers and Information in Engineering Conference, American Society of Mechanical Engineers, pp. V05CT08A043-V05CT08A043.

[23] Goldberg, M., 1942. “Linkages polyhedral”. National Mathematics Magazine, 16(7), pp. 323-332.

[24] Connelly, R., 1979. “The rigidity of polyhedral surfaces”. Mathematics Magazine, 52(5), pp. $275-283$. 


\section{Figure Caption List}

Figure 1. Construction of a Bricard linkage

Figure 2. Schematic of the type III Bricard linkage

Figure 3. Two collapsed configurations of the type III Bricard (a)-(b)

Figure 4. Dimensions of the quadrangle

Figure 5. Construction of a crinkle mechanism

Figure 6. Movement process of a crinkle linkage (a)-(c)

Figure 7. Limit positions of $A$ and $A^{\prime}$

Figure 8. Influence of $R$ on the collapsed configurations

Figure 9. Influence of $\theta$ and $r$ on the collapsed configurations (a)-(b)

Figure 10. Influence of $l_{A}$ and $l_{A}^{\prime}$ on the collapsed configurations (a)-(b)

Figure 11. Simulation of inverse calculation

Figure 12. Two Bricard linkages linked by scissor linkage (a)-(b)

Figure 13. The compact and deployed configuration of the network linked with scissor linkage (a)-(b)

Figure 14. Working principle of the parallelogram mechanism (a)-(c)

Figure 15. Assembly comprised of Bricard linkages and a parallelogram mechanism (a)-(b)

Figure 16. Formed rectangle corresponding to variable $\theta$

Figure 17. Case study I (a)-(b)

Figure 18. Bricard linkages in case study II

Figure 19. Case study II (a)-(d) 